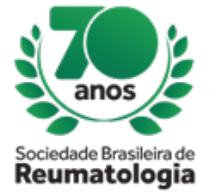

\title{
ACUTE FEBRILE NECK PAIN AND CROWNED DENS SYNDROME: A CASE REPORT AND REVIEW OF LITERATURE.
}

Adriane Tedgue Souza da Paz (Hospital Professor Edgard Santos, Salvador, BA, Brasil), Pedro Herrera Camargo (Hospital Professor Edgard Santos, Salvador, BA, Brasil), Gustavo Luiz Behrens Pinto (Hospital Professor Edgard Santos, Salvador, BA, Brasil), Mittermayer Barreto Santiago (Hospital Professor Edgard Santos, Salvador, BA, Brasil), Anna Paula Mota Duque Sousa (Hospital Professor Edgard Santos, Salvador, BA, Brasil)

\section{BACKGROUND}

Calcium pyrophosphate deposition disease (CPPD) is a systemic inflammatory arthropathy that affects 4$7 \%$ of the adult population (1). In despite of rare cases being described in the literature, crowned dens syndrome (CDS) has a prevalence of $12.5 \%$ in CPPD patients and $2 \%$ of them have acute neck pain $(1,2)$. We report a case of febrile acute neck pain secondary to CDS.

\section{CASE REPORT}

A 63-year-old woman with previous diagnosis of CPPD started having cervical pain, limited mobility and fever, accompanied by neck stiffness, for the last six weeks. She also had left knee arthritis and low back pain. Besides knee effusion and neck stiffness the rest of physical examination was unremarkable. Laboratory workup revealed leukocytosis, elevated platelets count and increased reactive $C$ protein. Serology for viral hepatitis, HIV and syphilis was negative; radiograph of knees showed chondrocalcinosis; Blood culture resulted positive for staphylococcus aureus; Transesophageal echocardiogram was normal. A cervical computed tomography scan demonstrated dense calcification in the form of a halo around the odontoid process, in the atlantoaxial joint, which is characteristic of CDS (Figure 1 to 4 ). The patient was treated with diclofenac and colchicine, with great improvement of the cervicalgia, fever, joint mobility and peripheral articular symptoms. She also received intravenous oxacillin for bloodstream infection.

\section{CONCLUSION}

CDS is an uncommon manifestation of CPPD. First described in 1980, it is characterized by cervicalgia, fever and neck stiffness. From the pathophysiological point of view, there is a deposition of calcium pyrophosphate (3). In the present case, it was probably associated with bloodstream infection. Differential diagnosis with meningitis is crucial. The gold standard diagnostic test is cervical spine tomography and the patients commonly have a good response to the treatment, as it was showed in this case. 